\title{
Comparing the Effect of Open and Closed Kinetic Chain Exercises in Patients Suffering From Patellofemoral Pain Syndrome
}

\author{
Zahra Firoozkoohi Moghadam ${ }^{1}$ Ahmad Ebrahimi Atri ${ }^{*}$, Seyed Ali Akbar Hashemi Javaheri ${ }^{3}$ \\ ${ }^{1}$ Master Student of Sport Injuries and Corrective Exercise, International Branch of Ferdowsi University of Mashhad, \\ Mashhad, Iran \\ ${ }^{2}$ Associate Professor of Exercise Physiology, Faculty of Physical Education and Sport Sciences, Ferdowsi University of \\ Mashhad, Mashhad, Iran \\ ${ }^{3}$ Associate Professor of Therapeutic Exercises, Faculty of Physical Education and Sport Sciences, Ferdowsi University of \\ Mashhad, Mashhad, Iran
}

*Correspondence to

Ahmad Ebrahimi Atri;

Email: atri@um.ac.ir

Received July 31, 2016 Revised August 10, 2016

Accepted August 31, 2016 Published online September 26, 2016

Please cite this article as follows: Firoozkoohi Moghadam Z, Ebrahimi Atri A, Hashemi Javaheri SAA. Comparing the effect of open and closed kinetic chain exercises in patients suffering from patellofemoral pain syndrome. Int J Basic Sci Med. 2016;1(2):5357. doi:10.15171/ ijbms.2016.12.

\begin{abstract}
Introduction: Patellofemoral pain syndrome is one of the most prevalent problems in physically active people. The syndrome is a multifactorial disorder with different therapeutic approaches. Conservative treatment of the syndrome has been a crucial matter in different studies. Quadriceps muscle strengthening exercise is a recommended approach which is performed in forms of open and closed kinetic chain exercises. This study was designed to compare the efficacy of these exercises on this syndrome.

Methods: Totally 30 women suffering from the syndrome aged 30-40 were randomly allocated into three groups: open, and closed kinetic chain exercises, and control group, as groups $A$, B, and C, respectively. Groups A and B participated in 28 sessions of exercises in a period of four weeks and group $\mathrm{C}$ did not do any exercise in this period. Before and after the period, pain and function of the participants were assessed using visual analog scale (VAS) and Kujala patellofemoral scale, respectively. Data were analyzed using independent $t$ test, dependent $t$ test, and univariate variance analysis, while significance of data was determined at $P<0.05$.

Results: The study indicated that open kinetic chain exercises (group A) for 4 weeks brought significant changes in relieving the pain along with improving the function $(P=0.001)$. Likewise, it was revealed a significant pain relief as well as function improvement in group $\mathrm{B}(P=0.001)$. Comparing two groups illustrated that benefits of open kinetic chain exercises outweigh closed ones $(P=0.001)$.

Conclusion: Overall, both exercises were efficient in relieving pain as well as improving function, however open kinetic chain exercises were more contributory.

Keywords: Patellofemoral pain syndrome, Open kinetic chain exercises, Closed kinetic chain exercises, Pain, Function, PFPS.
\end{abstract}

\begin{abstract}
Introduction
Patellofemoral pain syndrome is one of the most prevalent injuries in the knees, and is one of the most frequent orthopedic complaints in clinical fields. ${ }^{1,2}$ This multifactorial syndrome is known by a pain in knee which is increased by activities that exert pressure on the patellofemoral joint such as climbing, stepping down the stairs, squatting, and long periods of sitting..$^{3-5}$ Since there are a wide variety of symptoms, making a definite diagnosis is pretty difficult. Malalignment of the lower extremity, increasing $\mathrm{Q}$ angle, muscular disequilib-
\end{abstract}

rium, decrease in the strength of vastus medialis oblique muscle, and ultimately overuse are some of the contributors of the disease. Although surgical procedures have been used successfully for these patients, conservative interventions are used widely especially in the first steps of the treatment. ${ }^{6}$ This kind of treatment entails $80 \%-90 \%$ of all.

On the other hand, since there are plenty of biomechanical factors relating to this problem, ${ }^{8,9}$ various conservative interventions such as exercise therapy, taping, brace, foot prostheses, soft tissue manipulation, and

Copyright (C) 2016 The Author(s); Published by Zabol University of Medical Sciences. This is an open-access article distributed under the terms of the Creative Commons Attribution License (http://creativecommons.org/licenses/by/4.0), which permits unrestricted use, distribution, and reproduction in any medium, provided the original work is properly cited. 
acupuncture have been suggested and evaluated for the treatment of this syndrome..$^{10}$

Earlier studies reported satisfactory results regarding the exercise therapy in these patients. ${ }^{5,11-15}$ Such exercises are widely accepted for relieving pain and improving joint function. ${ }^{6}$ Frye et al indicated in a systematic review that despite reported effectiveness about these exercises in improving joint function and diminishing pain, there is no consensus about the most effective therapeutic approach as well as the best method of exercise therapy. ${ }^{5}$

One of the therapeutic approaches for this syndrome is quadriceps muscle strengthening exercises. Open and closed kinetic chain exercises are some examples among many different strategic approaches for quadriceps muscle strengthening. ${ }^{16,17}$ Chain exercises activate different parts of the extremity that provides situations which result in pressure production, extremity fixation, and ultimately pressure transport to the end of extremity. ${ }^{18}$ Close chain exercises are multiple articular movements in which end of the extremity is fixed and occasionally related to weight bearing. By stark contrast, in open chain exercises, end of the extremity is not fixed and there is no weight bearing exercise. ${ }^{19}$ Witvrouw et al illustrated that there is no significant differences between two approaches. They concluded that both exercises are effective in muscle function improvement as well as pain alleviation, ${ }^{20}$ however, Collins et al recommended closed kinetic chain exercises for just short-term periods. ${ }^{10}$

Considering the fact that there is no consensus about the best and most effective exercises for treatment of patellofemoral pain syndrome, and high prevalence of the disease in our community, this study aimed to compare two methods of kinetic chain exercises-open and closedregarding their efficacy in alleviating pain and improving joint function in this syndrome.

\section{Methods}

This case-control study was done on females who referred to a physiotherapy clinic diagnosed with patellofemoral pain syndrome in Zabol, Iran. Among these patients, 30 subjects were allocated randomly into 3 groups: group A for performing open kinetic chain exercises (10 subjects), group B for closed kinetic chain exercises (10 subjects), and group $\mathrm{C}$ as control group (10 subjects). Inclusion criteria were: being aged between 30 and 40, absence of tendon and/or meniscus problem in knees (based on the report of the physician), minimum visual analog scale (VAS) score of three as well as Kujala score of 50, positive grind test along with other clinical tests, and confirmation of diagnosis of patellofemoral pain syndrome by a specialist.

All of the participants filled a questionnaire out about demographic data and signed a written consent. Then, severity of pain in patients was measured using VAS. Function of the joints was assessed using Kujala disability specific questionnaire as well. At next step, groups A and $B$ were treated by 28 sessions of treatment during a period of 4 weeks. Each session lasted about 45-60 minutes. The control group (group C) continued their daily routine as previous without any special physical activity. After termination of this period, all of the cases and controls filled out abovementioned questionnaire again. All of the three groups had the same medication protocol. They were trained for their daily activity, diet and sport. It was emphasized that the patients follow this daily routine.

Kujala questionnaire was introduced first by Kujala in 1993 for evaluating function of the knee in patients who suffer from patellofemoral pain syndrome. The questionnaire consists of 10 objective and subjective questions with minimum score of zero (loss of function) and maximum of 100 (perfect function). This questionnaire was translated into Persian by Negahban et al. ${ }^{21}$ Its validity and reliability were confirmed with intragroup correlation scale in test and retest $(\mathrm{ICC}=0.96)$ and Cronbach $\alpha$ of $81 \%$, respectively.

VAS was used to measure perception of the pain. The scale is a 10 centimeter horizontal band in which one extreme is zero (no pain) along with other extreme which is 10 (the most severe pain). Patients were asked to mark one point in this 10 centimeter line based on the severity of pain. This scale is the most reliable scaling system for measuring pain that is widely used in pain related researches. Its validity and reliability is confirmed.

Regarding the open and closed kinetic chain exercises, both of the groups A and B had warm up and cool down exercises for about 5-10 minutes. The exercises in the first half of sessions were much easier than the second half with less severity and duration as well as repetition. Therefore, patients had much more time for rest in early stages. Gradually, exercises were more severe considering increased patients' ability and more pressure. In first two weeks each main exercise was repeated 10 times, while the contractions lasted 10 seconds. Then in last two weeks, time of each contraction was increased to 20 seconds.

Data were analyzed using SPSS version 22.0. Independent $t$ test, dependent $t$ test, and univariate variance analysis were conducted in significance level of less than 0.05 .

\section{Results}

Demographic characteristics of the patients are shown in Table 1. There were no significant difference among the groups regarding the age, height, and weight.

Results indicated that pain relief and joint function improvement was significant in groups $\mathrm{A}$ and $\mathrm{B}$ with open and closed kinetic chain exercises $(P=0.001)$. Comparing two groups illustrated that benefits of open kinetic chain exercises outweigh closed ones in relieving pain and improving joint function $(P=0.001)$ (Table 2$)$.

\section{Discussion}

The study showed that a period of open or closed kinetic chain exercises on quadriceps muscle has a significant effect on relieving pain and improving joint function. Comparing two methods of exercises illustrated significant higher efficiency for open kinetic chain exercises.

Results of previous studies are compatible with the find- 
Table 1. Mean and Standard Deviation of Age, Height, and Weight in Three Groups of Open, Closed, and Control

\begin{tabular}{|c|c|c|c|c|}
\hline Variable & Open Kinetic Chain Group & Closed Kinetic Chain Group & Control Group & $P$ Value \\
\hline Age (y) & $39.60 \pm 3.40$ & $40.50 \pm 2.36$ & $38.90 \pm 3.07$ & 0.73 \\
\hline Height $(\mathrm{cm})$ & $163.50 \pm 2.83$ & $162.10 \pm 4.45$ & $160.30 \pm 5.53$ & 0.84 \\
\hline Weight (kg) & $69.61 \pm 6.78$ & $66.39 \pm 3.31$ & $69.30 \pm 5.53$ & 0.60 \\
\hline $\mathrm{BMI}\left(\mathrm{kg} / \mathrm{m}^{2}\right)$ & $26.33 \pm 1.67$ & $25.20 \pm 1.32$ & $25.46 \pm 1.41$ & 0.62 \\
\hline
\end{tabular}

Abbreviation: BMi, body mass index.

Table 2. Comparing Mean of Some Pain and Function Indexes in Groups of Open, Closed, and Control

\begin{tabular}{|c|c|c|c|c|c|c|c|}
\hline \multirow{2}{*}{ Variable } & \multirow{2}{*}{ Group } & \multicolumn{2}{|c|}{ Stage } & \multicolumn{2}{|c|}{ Intragroup Changes } & \multicolumn{2}{|c|}{ Intergroup Changes } \\
\hline & & Pre-test & Post-test & $t$ & $\boldsymbol{P}$ & $\boldsymbol{F}$ & $\boldsymbol{P}$ \\
\hline \multirow[t]{3}{*}{ Pain } & Open kinetic chain & $7.90 \pm 0.99$ & $4.5 \pm 1.08$ & -20.82 & 0.001 & 70.54 & 0.001 \\
\hline & Closed kinetic chain & $5.9 \pm 0.87$ & $4.3 \pm 0.94$ & -9.79 & 0.001 & & \\
\hline & Control & $4.3 \pm 1.15$ & $4.1 \pm 0.87$ & 0.68 & 0.50 & & \\
\hline \multirow[t]{3}{*}{ Function } & Open kinetic chain & $65.7 \pm 4.78$ & $77.9 \pm 5.50$ & -19.39 & 0.001 & 105.07 & 0.001 \\
\hline & Closed kinetic chain & $63.5 \pm 3.43$ & $69.8 \pm 2.78$ & -9.94 & 0.001 & & \\
\hline & Control & $61 \pm 3.82$ & $60.1 \pm 3.41$ & 1.36 & 0.2 & & \\
\hline
\end{tabular}

ings of our study. Witvrouw et $\mathrm{al}^{22}$ evaluated effects of long term open and closed kinetic chain exercises on patients with patellofemoral pain. They reported that both exercises resulted in sustained positive effects on patients. Similarly, Minoonejad et $\mathrm{al}^{23}$ showed satisfactory results about relieving pain and increasing knee flexion in stepping stairs up and down. One of the possible reasons for this similarity in the results might be using the same exercise protocols.

In contrast, the results of Peeler et $\mathrm{al}^{24}$ study showed different results. They studied effects of exercise therapy on the function of patellofemoral pain syndrome. Some of the possible reasons might be different duration of therapeutic period ( 3 weeks in the study of Peeler et al), number of sessions per week, and sample size.

As stated earlier, in this study, two methods of open and closed kinetic chain exercises were evaluated for their efficiency in alleviating pain along with improving joint function in patients with patellofemoral pain syndrome. ${ }^{25}$ One of the possible reasons for alleviating pain and improving function with open and closed kinetic chain exercises is the fact that strengthening quadriceps muscle especially vastus medialis obliquus (VMO) muscle results in suitable and coordinated contraction of the muscle; therefore it would correct tracking of the patella and diminish forces on the knees. This would lead to joint function improvement and pain decrease. ${ }^{26}$ In order to confirm this hypothesis, it should be emphasized that mechanism of pain in patellofemoral pain syndrome is weakness of quadriceps muscle along with muscle imbalance especially in VMO muscle. ${ }^{27}$ Besides, agonistic contraction of hamstring and quadriceps muscles during closed kinetic chain contractions leads to better function of knee joint as well as tibiofemoral joint. ${ }^{28}$

Explaining possible mechanism of improving function and decreasing pain in open kinetic chain exercises, it should be considered that these types of exercises result in the isolation of quadriceps muscle; so it can be contracted solely and it can strengthen the muscle. ${ }^{29}$ Quadriceps strengthening improves joint function. Since deficiency in neuromuscular system is another main problem in $\mathrm{Pa}$ tellofemoral Pain Syndrome patients, ${ }^{30}$ another possible mechanism in effectiveness of open and closed kinetic chain exercises might be the effectiveness of these exercises in improving neuromuscular system of knee joint as well as in increasing proprioception in the joint.

Improving the neuromuscular system and muscle strengthening can mend pattern of muscle activity along with fixed stability of knee joint and ultimately can diminish forces on the joint. ${ }^{31}$

Increase in muscle spindle sensitivity would progress the afferent neurons' information to central nervous system about position of the joint. Such exercises can increase proprioception and joint function. ${ }^{32}$ These factors would improve joint function in patellofemoral pain syndrome patients.

Another result of the study was the fact that open kinetic chain exercises were more effective than close ones in alleviating pain as well as improving joint function in patellofemoral pain syndrome patients. Another study showed that closed kinetic chain exercises had more effect in increasing proprioception. ${ }^{33}$ As mentioned earlier open kinetic chain exercises improve solely contraction of quadriceps ${ }^{29}$ and this might be a possible reason for being more effective comparing to closed ones in our study.

Our study had some limitations. All the three groups were trained for their daily activity, diet, and sport and it was emphasized that the patients follow this daily routine. However, we could not follow these variables properly since our data reference was claim of the patients. Therefore we could not confirm that all of the groups were matched for their daily activity, diet and sport during 
the study period. Another limitation was that we did not evaluate the efficacy of the exercises based on the sex, age, body mass index (BMI), function score, Q angle, and severity of the pain in first visit. Authors suggest more work considering these variables and factors in their studies.

\section{Conclusion}

Two methods of open and closed kinetic chain exercises were effective in alleviating pain along with improving joint function in patellofemoral pain syndrome patients. Besides, in comparison of these two methods, open kinetic chain exercises had more positive effects rather than closed ones.

\section{Ethical Approval}

Ethical committee of Zabol University of Medical Sciences, Zabol, Iran, approved the study under the code number of zbmu.1.REC.1395.50.

\section{Competing Interests}

Authors declare that they have no competing interests.

\section{Acknowledgments}

Authors would like to thank all staffs and patients who helped them in this study.

\section{References}

1. Blond L, Hansen L. Patellofemoral pain syndrome in athletes: a 5.7-year retrospective follow-up study of 250 athletes. Acta Orthop Belg. 1998;64(4):393-400.

2. Wood L, Muller S, Peat G. The epidemiology of patellofemoral disorders in adulthood: a review of routine general practice morbidity recording. Prim Health Care Res Dev. 2011;12(2):157-164. doi:10.1017/ s1463423610000460.

3. Thomeé R, Augustsson J, Karlsson J. Patellofemoral pain syndrome. Sports Med. 1999;28(4):245-262. doi:10.2165/00007256-199928040-00003.

4. Baker V, Bennell K, Stillman B, Cowan S, Crossley $\mathrm{K}$. Abnormal knee joint position sense in individuals with patellofemoral pain syndrome. J Orthop Res. 2002;20(2):208-214. doi:10.1016/s0736-0266(01)00106-1.

5. Frye JL, Ramey LN, Hart JM. The effects of exercise on decreasing pain and increasing functionin patients with patellofemoral pain syndrome a systematic review. Sports Health. 2012;4(3):205-210.

6. Rothermich MA, Glaviano NR, Li J, Hart JM. Patellofemoral pain: epidemiology, pathophysiology, and treatment options. Clin Sports Med. 2015;34(2):313-327. doi:10.1016/j.csm.2014.12.011.

7. Post WR. Patellofemoral pain: results of nonoperative treatment. Clin Orthop Relat Res. 2005;436:55-9. doi:10.1097/01.blo.0000172306.97285.95.

8. Lankhorst NE, Bierma-Zeinstra SM, van Middelkoop M. Factors associated with patellofemoral pain syndrome: a systematic review. Br J Sports Med. 2013;47(4):193-206. doi:10.1136/bjsports-2011-090369.

9. Lankhorst NE, Bierma-Zeinstra SM, van Middelkoop M. Risk factors for patellofemoral pain syndrome: a systematic review. J Orthop Sports Phys Ther. 2012;42(2):81-A12. doi:10.2519/jospt.2012.3803.
10. Collins NJ, Bisset LM, Crossley KM, Vicenzino B. Efficacy of nonsurgical interventions for anterior knee pain. Sports Med. 2012;42(1):31-49. doi:10.2165/11594460000000000-00000.

11. Crossley K, Bennell K, Green S, Cowan S, McConnell J. Physical therapy for patellofemoral pain A randomized, double-blinded, placebo-controlled trial. Am J Sports Med. 2002;30(6):857-865. doi:10.1097/00005768-20010500100493.

12. Dutton RA, Khadavi MJ, Fredericson M. Update on rehabilitation of patellofemoral pain. Curr Sports Med Rep. 2014;13(3):172-178. doi:10.1249/jsr.0000000000000056.

13. Harvie D, O'Leary T, Kumar S. A systematic review of randomized controlled trials on exercise parameters in the treatment of patellofemoral pain: what works. J Multidiscip Healthc. 2011;4:383-392. doi:10.2147/jmdh.s24595.

14. Keays SL, Mason M, Newcombe PA. Individualized physiotherapy in the treatment of patellofemoral pain. Physiother Res Int. 2015;20(1):22-36. doi:10.1002/pri.1593.

15. Lack S, Barton C, Vicenzino B, Morrissey D. Outcome predictors for conservative patellofemoral pain management: a systematic review and meta-analysis. Sports Med. 2014;44(12):1703-1716. doi:10.1007/s40279014-0231-5.

16. Laprade J, Culham E, Brouwer B. Comparison of five isometric exercises in the recruitment of the vastus medialis oblique in persons with and without patellofemoralpain syndrome. J Orthop Sports Phys Ther. 1998;27(3):197-204.

17. Jonsson P, Alfredson H. Superior results with eccentric compared to concentric quadriceps training in patients with jumper's knee: a prospective randomised study. Br J Sports Med. 2005;39(11):847-850. doi:10.1136/ bjsm.2005.018630.

18. Escamilla RF, Fleisig GS, Zheng N, Barrentine SW, Wilk KE, Andrews JR. Biomechanics of the knee during closed kinetic chain and open kinetic chain exercises. Med Sci Sports Exerc. 1998;30(4):556-69. doi:10.1097/00005768199804000-00014.

19. Fagan V, Delahunt E. Patellofemoral pain syndrome: a review on the associated neuromuscular deficits and current treatment options. Br J Sports Med. 2008;42(10):789-795. doi:10.1136/bjsm.2008.046623.

20. Witvrouw E, Lysens R, Bellemans J, Peers K, Vanderstraeten G. Open versus closed kinetic chain exercises for patellofemoral pain a prospective, randomized study. Am J Sports Med. 2000;28(5):687-94. doi:10.1177/0363546503262187.

21. Negahban H, Pouretezad M, Yazdi MJS, et al. Persian translation and validation of the Kujala Patellofemoral Scale in patients with patellofemoral pain syndrome. Disabil Rehabil. 2012;34(26):2259-2263. doi:10.3109/0963 8288.2012.683480.

22. Witvrouw E, Danneels L, Van Tiggelen D, Willems TM, Cambier D. Open versus closed kinetic chain exercises in patellofemoral pain a 5 -year prospective randomized study. Am J Sports Med. 2004;32(5):1122-1130.

23. Minoonejad H, Rajabi R, Ebrahimi-Takamjani E, et al. Combined open and closed kinetic chain exercises for patellofemoral pain syndrome: a randomized controlled trial. World J Sport Sci. 2012;6(3):278-285.

24. Peeler J, Anderson JE. Effectiveness of static quadriceps stretching in individuals with patellofemoral joint pain. Clin J Sport Med. 2007;17(4):234-241. doi:10.1097/ 
jsm.0b013e3180f60afc.

25. Ferber R, Kendall KD, Farr L. Changes in knee biomechanics after a hip-abductor strengthening protocol for runners with patellofemoral pain syndrome. J Athl Train. 2011;46(2):142-149.

26. Yalfani A, Raisi Z. Comparison of two methods quadriceps muscle strengthening on land and in the water on pain, function, static and dynamic balance in females with patellofemoral pain syndrome. Sport Med Stud. 2013;5(13):91-108.

27. Mazloum V, Sahebozamani M. A Review Study on Various Conservative Management Strategies for Patellofemoral Pain Syndrome: What Is the Best Intervention? Journal of Kerman University of Medical Sciences. 2016;23(1):116136.

28. Worrell TW, Crisp E, LaRosa C. Electromyographic reliability and analysis of selected lower extremity muscles during lateral step-up conditions. J Athl Train. 1998;33(2):156.
29. Fehr GL, Junior AC, Cacho ÊWA, de Miranda JB. Effectiveness of theopen kinetic chain and closed kinetic chain in the treatment of patellofemoral pain syndrome. Rev Bras Med Eporte. 2006;12(2):66-70.

30. Callaghan M, McCarthy C, Oldham J. Electromyographic fatigue characteristics of the quadriceps in patellofemoral painsyndrome. Man Ther. 2001;6(1):27-33.

31. Roberts D, Friden T, Stomberg A, Lindstrand A, Moritz U. Bilateral proprioceptive defects in patients with a unilateral anterior cruciate ligament reconstruction: a comparison between patients and healthy individuals. J Orthop Res. 2000;18(4):565-571. doi:10.1002/jor.1100180408.

32. Edin BB. Cutaneous afferents provide information about kneejointmovementsinhumans.JPhysiol.2001;531(1):289297. doi:10.1111/j.1469-7793.2001.0289j.x.

33. Prentice WE, Kaminski TW. Rehabilitation techniques for sports medicine and athletic training. New York: McGrawhill; 2004. 